\title{
Liquid-Phase Peak Force Infrared Microscopy
}

\author{
Haomin Wang, ${ }^{1}$ Joseph M. González-Fialkowski, ${ }^{1}$ Wenqian $\mathrm{Li},{ }^{2}$ Qing Xie, ${ }^{1}$ Yan Yu, ${ }^{2}$ and Xiaoji \\ G. $\mathrm{Xu}^{* 1}$ \\ ${ }^{1}$ Department of Chemistry, Lehigh University, 6 E Packer Ave., Bethlehem, PA, 18015, United \\ States \\ ${ }^{2}$ Department of Chemistry, Indiana University, 800 E Kirkwood Ave, Bloomington, IN, 47405, \\ United States \\ *E-mail: xgx214@ lehigh.edu
}

\begin{abstract}
Detection of infrared photothermal response with atomic force microscopy (AFM) bypasses Abbe's diffraction limit in achieving chemical nano-imaging and spectroscopy. The peak force infrared (PFIR) microscopy is one of the emerging AFM-based infrared microscopies with excellent spatial resolution. Here, we develop the PFIR microscopy to operate in the liquid phase with the capability of controlling fluid compositions. The resulting liquid-phase peak force infrared (LiPFIR) microscopy enables non-destructive and label-free infrared imaging in the liquid on a series of soft matters with $<10 \mathrm{~nm}$ spatial resolution. We use LiPFIR to track the polymer surface reorganization in fluids and detect the product of click chemical reaction in the aqueous phase. As a biological application of LiPFIR, the budding site of yeast cell wall particles is imaged in water. The super-resolution, label-free, non-destructive chemical imaging and spectroscopy capabilities of LiPFIR will facilitate investigations of chemical compositions and transformations at the liquid/solid interface.
\end{abstract}




\section{Introduction}

While Abbe's diffraction limit restricts the spatial resolution of traditional IR microscopy to half of the wavelength of several microns ${ }^{1}$, the integration of atomic force microscopy (AFM) with IR radiation creates routes to achieve nanometer-scale spatial resolution. ${ }^{2,3}$ Although light detection through near-field scattering provides an optical route to bypass the diffraction limit, the mechanical detection of the IR induced actions by a sharp AFM probe offers a high-sensitivity route to achieve IR nanoscopy, particularly for soft matters, with simple instrumentations. ${ }^{4-6}$ The photothermal induced resonance (PTIR), ${ }^{7,8}$ resonantly enhanced infrared photo-expansion nanoscopy (REINS), ${ }^{9}$ photo-induced force microscopy (PiFM), ${ }^{5}$ and peak force infrared (PFIR) microscopy ${ }^{6}$ are increasingly popular representatives of AFM-based IR methods, offering IR imaging and spectroscopy with direct correspondence to the IR absorption of the sample. The PFIR microscopy, which operates with peak force tapping mode, combines the respective advantages of PTIR and PiFM to achieve a high spatial resolution of $<10 \mathrm{~nm}$, unambiguously photothermal signal generation mechanism, and non-destructiveness to the sample surface. PFIR also offers complimentary mechanical information channels, such as modulus and adhesion between the tip and sample, allowing the additional understanding of the sample beyond the IR absorption. ${ }^{6,10}$

On the flip side, another challenge is typically associated with IR microscopies — incompatibility with aqueous conditions. Water strongly attenuates the IR radiation, which makes the delivery and detection of IR in regular far-field microscopy extremely challenging in the aqueous phase. AFMbased IR microscopies provide a unique edge to address this challenge with the implementation of total internal reflection geometry. ${ }^{11-16}$ The evanescent IR field of the total internal reflection excites the vibrational modes of molecules and materials; the AFM tip probes the actions of the photothermal response excited by the evanescent field, providing spatial resolution and avoiding 
the attenuation loss of the optical detection. REINS and contact mode PTIR have been demonstrated in the fluid phase on polymers and proteins with $20 \sim 25 \mathrm{~nm}$ spatial resolution. ${ }^{11,12}$ However, the PTIR and REINS are based on contact mode AFM, which is prone to the scratching of the sample surface, particularly in the liquid phase. The constant contact between the tip and sample and lateral force in the scan lead to deformations and damages of the sample even in a short time (see Supplementary Figure S1). The s-SNOM technique, which is based on tapping mode AFM, does not suffer from the lateral force. s-SNOM with total internal reflection and bottom-up illumination have been applied in water. ${ }^{14,15}$ However, s-SNOM suffers from the detection loss from water absorption after the near-field scattering. Moreover, s-SNOM setup has a higher instrumental complexity and often requires two sets of light sources for IR imaging and spectroscopy.

The peak force tapping mode has been proven to operate stably in the liquid phase without sample damage for living cells ${ }^{17}$ and single membrane proteins. ${ }^{18}$ High-resolution AFM images of peak force tapping mode are routinely achieved in the liquid phase. PFIR microscopy inherits the advantages of peak force tapping mode. It is suitable for liquid phase applications on delicate soft matters while preserving the integrity of the sample surface. The non-destructiveness of the PFIR microscopy makes it an attractive method to study physical transformations and chemical reactions in the fluid phase. In this article, we develop the liquid phase peak force infrared (LiPFIR) microscopy with the ability to adjust the types of fluids on-demand to initiate physical transformations and chemical reactions. IR imaging and spectroscopy on polymer surface reorganization and click chemistry are demonstrated in situ. The spatial resolution of LiPFIR is found to be $<10 \mathrm{~nm}$ in the fluid conditions on protein fibrils. Finally, a biological sample of two 
zymosan particles in the budding process is imaged in water, revealing the presence of proteins around the budding site.

\section{Results and Discussion}

\section{Operational Principle and Design of LiPFIR Microscopy}

LiPFIR microscopy inherits the operational principle of PFIR microscopy ${ }^{6}$ with the addition of evanescent field delivery. It uses an AFM cantilever to mechanically detect photothermal expansions of the sample submerged in the liquid. The challenge of high attenuation of IR in water is overcome by the utilization of the total internal reflection geometry with a germanium prism. Because the photothermal signal of the IR absorption is mechanically detected in LiPFIR, the would-be propagation loss of the IR signal in the optical detection after the light-matter interaction is bypassed. Figure 1a illustrates the total internal reflection geometry used in the LiPFIR microscopy. A gold AFM tip is placed above the germanium surface, and IR pulses are guided from the bottom at an incident angle of $20^{\circ}$ to enable the total internal reflection. A finitedifference time-domain (FDTD) simulation of such a scheme is displayed in Figure 1b, where an intensity enhancement of $5.2 \times 10^{2}$ is achieved at the germanium surface underneath the AFM tip. Spatial confinement with the full width at half maximum (FWHM) of only $25 \mathrm{~nm}$ is observed, which is vital for AFM-based super-resolution imaging. This tip-enhanced field excites local sample molecules. The absorbed energy is then converted into photothermal expansions, which are mechanically detected by the motion of AFM cantilever. The peak force tapping (PFT) mode is used in LiPFIR. In PFT mode, the distance between the tip and sample oscillates at a low frequency of a few $\mathrm{kHz}$. The tip momentarily contacts the sample surface for tens of microseconds and detaches afterward in every PFT cycle. ${ }^{19,20}$ The shear force that is built up in the lateral scan 
is released when the tip and the sample detach, avoiding the stick-slip motion of the contact mode AFM. ${ }^{21}$ Unlike tapping mode AFM, the feedback of PFT is based on the maximal cantilever deflection during the tip-sample contact, which is not affected by the hydrodynamic drag force of the fluid.

Figure 1c illustrates the LiPFIR apparatus consisting of a frequency tunable IR source, a liquidphase compatible AFM, and a germanium prism. A quantum cascade laser (QCL, MIRcat-QT, DRS Daylight Solutions) provides tunable IR laser pulses up to $1 \mu$ s duration with adjustable repetition rates. The beam from QCL is firstly expanded by a reflective telescope (BE04, Thorlabs) and then coupled into a germanium prism using a germanium lens with a $2.5-\mathrm{cm}$ focal length. A PFT-enabled AFM (Bioscope Catalyst, Bruker) operates on the germanium prism with a metalcoated probe and the PFT frequency of $1 \mathrm{kHz}$. A transistor to transistor logic (TTL) waveform from the phase lock loop (PLL) at the PFT frequency is routed to a function generator in the burst mode to trigger the QCL to emit a train of IR pulses. The timing of the emission is set to the moment when the tip and sample are in dynamic contact. The deflection of the cantilever is read out by a built-in quadrant photodiode in AFM. A perfusion chamber is sealed by an O-ring to enclose both the cantilever and the sample. The perfusion chamber has a fluid inlet and an outlet, enabling changing the fluid composition to initiate physical transformations and chemical reactions.

The calculated relationship between the Z-component of the evanescent field $\mathrm{E}_{\mathrm{Z}}$ and the incident angle $\theta$ is plotted in Figure $1 \mathrm{~d} .{ }^{22}$ As the incident angle approaches the critical angle $\theta_{\mathrm{c}}$ (which is calculated to be $\left.19^{\circ}\right), \mathrm{E}_{Z}$ is significantly enhanced. ${ }^{11}$ In practice, an incident angle of $21^{\circ}$ is used in LiPFIR experiments, which is slightly larger than the critical angle, and large enough to ensure total internal reflection for all mid-IR frequencies. As Figure 1b indicates, the metallic AFM tip 
further amplifies the evanescent field, and the laser-induced photothermal expansion will occur at a nanometre-size hotspot underneath the tip, providing a high spatial resolution.

Figure 1e shows an experimentally collected cantilever deflection curve that contains photothermal expansion signals caused by IR absorptions. The cantilever oscillations are slightly enhanced by matching the repetition rate of the pulse train to one of the contact resonances of the cantilever. Figure 1f shows the zoomed-in region of the laser-induced oscillations. A polynomial fit $\left(4^{\text {th }}\right.$ order $)$ is performed to remove the slow varying curvature of the cantilever deflection in Figure $1 \mathrm{f}$ to retrieve pure oscillations at the repetition frequency of the laser pulse (Figure 1g). This procedure

bypasses the self-reference scheme of the original PFIR microscopy, ${ }^{6,23}$ and doubles the signal acquisition speed. The amplitude of the cantilever oscillations is extracted with a fast Fourier transform. The resulting peak corresponding to the laser repetition rate is integrated and used as the LiPFIR signal (Figure 1h). IR nano-imaging is achieved by scanning the AFM tip over the sample surface while registering the LiPFIR signal with a fixed laser frequency; nanospectroscopy is obtained by parking the AFM tip at a desirable location on the sample and sweeping the frequency of the QCL.

\section{Identification of Nanoscale Domains of Polymer Blends and Temporal Tracking of Surface Reorganization by LiPFIR}

Polymer blends formed by spontaneous phase separation serve as a model of soft matter to demonstrate the imaging and spectroscopy capability of LiPFIR microscopy. Figure 2a shows the topography of the polymer blend film (250-nm thick) formed by polystyrene (PS) and polymethyl methacrylate (PMMA) measured in heavy water. Two types of domains are noticeable in the topography: higher circular islands and a lower matrix region. Subjected to preparation conditions, 
spin-coated PS:PMMA blends can take different phase-separated geometries that cannot simply be distinguished from the topography. IR nano-imaging is required to perform chemical identification. To do so, the incident laser of the LiFPIR microscopy is tuned to IR frequencies that correspond to vibrational resonances of PS and PMMA, respectively. The resulting IR images are displayed in Figure 2b-c. At $1492 \mathrm{~cm}^{-1}$, PS domains exhibit a larger photothermal expansion signal as aromatic $\mathrm{C}=\mathrm{C}$ bonds absorb strongly at this frequency. At $1725 \mathrm{~cm}^{-1}$, PMMA domains are highlighted due to the absorption of the carbonyl $\mathrm{C}=\mathrm{O}$ vibrations. Figures $2 \mathrm{~b}$-c allow chemical identification of the domain compositions of polymer submerged in the liquid phase: the higher islands in the PS:PMMA blend polymer film are PMMA domains, and the lower matrix is the PS domain.

Point spectra collected with LiPFIR are shown in Figure 2d. When the AFM tip is fixed on circular islands, the averaged spectrum shows IR signatures of PMMA. Besides a strong carbonyl absorption peak at $1725 \mathrm{~cm}^{-1}$, there are also two peaks at $1380 \mathrm{~cm}^{-1}$ ( $\alpha$-methyl group vibration) and $1445 \mathrm{~cm}^{-1}$ (methyl C-H vibrations). In comparison, the averaged spectrum obtained from the PS domain shows absorption peaks at $1450 \mathrm{~cm}^{-1}, 1495 \mathrm{~cm}^{-1}$, and $1601 \mathrm{~cm}^{-1}$, all of which are from aromatic $\mathrm{C}=\mathrm{C}$ vibrations of PS molecules. The distinctive spectra for PS and PMMA nanodomains indicate the unambiguous chemical sensitivity of LiPFIR. Note that the carbonyl peak at $1725 \mathrm{~cm}^{-}$ ${ }^{1}$ of PMMA is also present in the PS domain, which is likely caused by bulk absorption of PMMA. The same effect was also observed in PS-b-PMMA copolymers with standard air phase PFIR, ${ }^{6}$ as well as in PiFM in the air. ${ }^{5}$ These results demonstrate that LiPFIR is capable of distinguishing multi-component polymer domains through both IR imaging and spectroscopy. The signal-tonoise ratio (relative peak height divided by the standard deviation of the background) of the 1725 $\mathrm{cm}^{-1}$ peak measured at the PMMA domain is calculated to be 45 . 
In the organic solvent of ethanol, PS:PMMA polymer film swells and rearranges motifs toward thermodynamic equilibrium. ${ }^{24}$ The LiPFIR microscopy allows monitoring this effect in situ with chemical imaging of polymer domains over time. In this experiment, we inject ethanol into the perfusion cell and observe the surface reorganization of PS:PMMA blend film through LiPFIR imaging. Figures $2 \mathrm{e}-\mathrm{h}$ show the surface reorganization on a $5 \times 5 \mu \mathrm{m}^{2}$ area of the polymer blend film over time. At the beginning (Figure 2e), the IR imaging at $1725 \mathrm{~cm}^{-1}$ shows that small islands are PMMA domains, and the matrix that surrounds the islands is the PS domain. As time goes by, small PMMA domains swell, because PMMA has a high affinity with ethanol. ${ }^{25}$ PMMA domains also coalesce into each other to form larger domains, as demonstrated in LiPFIR images in Figures $2 \mathrm{f}$ and $2 \mathrm{~h}$. For the polymer morphology in Figures $2 \mathrm{f}-\mathrm{h}$, the larger PMMA domain from the coalescence of small domains has elevated surface height from AFM topography (marked by white dashed enclosures in Figure 2h). The swelling process of single PMMA domains also enhances the LiPFIR signal. One example of the single PMMA domain swelling is shown in Figure $2 \mathrm{i}-\mathrm{j}$, where the small PMMA domain in the bottom left corner of the map increases its height by $20 \mathrm{~nm}$ and exhibits a larger IR absorption, after immersed in ethanol for $99 \mathrm{~min}$.

The driving force behind the surface reorganization of PMMA: PS film in ethanol is the difference in intermolecular forces and the resulting thermodynamics. Our observation shows that affinity exists between PMMA and ethanol, because the PMMA islands swell in ethanol. Also, the presence of ethanol shifts the thermodynamic equilibrium of PMMA:PS blend, causing PMMA domains to coalesce into each other when submerged in ethanol. This finding is consistent with a previous study of the solubility of PMMA and PS in ethanol. ${ }^{25}$ Our in situ IR imaging captures the onset of the physical transformation of the PS:PMMA polymer film in ethanol, which may lead to 
the eventual peeling of the polymer film from the prism surface. LiPFIR is a suitable tool to study the swelling process of the polymer films in fluids.

\section{Investigation of Reaction Sites of Click Chemistry}

Click chemistry offers a reliable way to synthesize chemicals with functional groups through the cycloaddition between azides and alkynes in water. ${ }^{26}$ In click chemistry, one of the most common strategies is copper-catalyzed azide-alkyne cycloaddition (CuAAC), where copper(I) is utilized as the catalyst. ${ }^{27} \mathrm{CuAAC}$ has been utilized widely in organic synthesis, ${ }^{27}$ polymer chemistry, ${ }^{28}$ medical applications, ${ }^{29}$ and bioconjugation labeling. ${ }^{30}$ Spatially detecting the location of the CuAAC process at the nanoscale provides indications to the site-specific reactivity, which is important in heterogeneous materials and complex biological systems. Here, we utilize the LiPFIR microscopy to pinpoint reaction sites of the heterogeneous (solid/liquid) $\mathrm{CuAAC}$ in water.

Figure $3 \mathrm{a}$ illustrates the reaction process of the CuAAC between the azide-terminated PMMA (PMMA-N3) and the alkyne-functionalized biotin through polyethylene glycol chains (biotinPEG-alkyne). Similar to Figure 2, PS:PMMA-N3 blend mixture is spin-coated on the prism surface

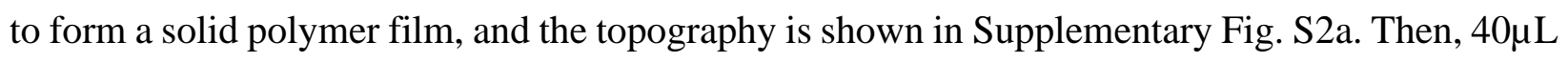
of the reaction solution containing biotin-PEG-alkyne, $\mathrm{CuSO}_{4}$ and $\mathrm{C}_{6} \mathrm{H}_{7} \mathrm{NaO}_{6}$ is dropped on the polymer to start the CuAAC reaction. To examine the change of polymer morphology induced by the CuAAC, the prism surface is rinsed with water after $90 \mathrm{~min}$ and air-dried. The polymer surface is then examined by the regular PFIR in the air in Supplementary Fig. S3. Several protrusions are found in the topography, which are proved to be $\mathrm{CuAAC}$ reaction sites through IR imaging. The PFIR spectrum collected from one reaction site shown in Figure $3 g$ exhibits a strong absorption peak of PEG at $1100 \mathrm{~cm}^{-1}$, confirming the existence of biotin-PEG-alkyne at reaction sites. 
Then, LiPFIR is used to locate the CuAAC reaction site in water in situ. After starting the reaction, the same sample area is scanned by LiPFIR intermittently without drying and LiPFIR images at $1100 \mathrm{~cm}^{-1}$ are captured. After 24 hours, a small protrusion area ( $200 \mathrm{~nm}$ in diameter) is located on the PMMA-N3 domain in Figure 3b. In Figure 3c, the adhesion map shows this protrusion area is likely extraneous. Figure $3 \mathrm{~d}$ illustrates four bright spots at $1100 \mathrm{~cm}^{-1}$ around the protrusion area, while under the PMMA-N3 absorbing frequency of $1160 \mathrm{~cm}^{-1}$ in Figure 3e, these spots remain dark. Two point spectra displayed in Figure $3 \mathrm{f}$ are collected from one of four spots and unreacted PMMA-N3 surface. A clear signature of PEG absorption at $1100 \mathrm{~cm}^{-1}$ is observed for the reaction site, while for the unreacted PMMA-N3, only the peak at $1160 \mathrm{~cm}^{-1}$ is present. The signal to noise ratio of the $1160 \mathrm{~cm}^{-1}$ peak for the PMMA-N3 domain in water is estimated to be 37 . From a crosssectioning profile of one hotspot in Figure 3d, the detection limit of LiPFIR is estimated to be $10^{3}$ biotin-PEG-alkyne molecules (Supplementary Fig. S4). While the air-phase PFIR provides the IR spectrum of the reaction product with excellent quality (Figure 3g), LiPFIR is capable of locating and distinguishing the $\mathrm{CuAAC}$ reaction site in operando, without drying the sample or interrupting the reaction. The joint usage of LiPFIR with CuAAC reaction will facilitate the biological investigations that use tags with click chemistry to pinpoint objects of interest at solid/liquid interface.

\section{Quantification of Spatial Resolution of LiPFIR}

Bovine serum albumin (BSA) is widely used as a model protein in many biological processes and applications. ${ }^{31,32}$ PTIR has been utilized in revealing the secondary structures of proteins. ${ }^{12,33}$ Here, we use LiPFIR to perform nano-imaging of the BSA sample in water. At room temperature, BSA proteins tend to form fibril structures in solution. ${ }^{34,35}$ To immobilize the BSA, the germanium surface is silanized with APTES (see Methods and Materials). AFM topography of a segment of a 
BSA fibril in $0.1 \times$ PBS buffer is shown in Figure 4a. This single BSA fibril is $150 \mathrm{~nm}$ in height and $350 \mathrm{~nm}$ in width. LiPFIR images at $1630 \mathrm{~cm}^{-1}$ (amide I band) are shown in Figure 4b-d in water and heavy water environments. In water, a large IR signal along the BSA fibril is observed, which distinguishes the protein fibril from the substrate. The spotty signals on the substrate are observed at interstices among the rough surface, where hot spots are created by strong field enhancements. In heavy water, amide I band IR absorption is present in the fibril. Another observation from Figure $4 \mathrm{c}$ is that a small region at the left terminal of the BSA fibril exhibits much reduced IR signal at $1630 \mathrm{~cm}^{-1}$. Such a difference in IR responses indicates a change of the protein secondary structure.

LiPFIR preserves the surface integrity of the sample during the measurement, and allows for repeated measurements of the same region while changing the composition of the fluid. The topography of the BSA fibril stays the same after 8 hours of scans (Supplementary Fig. S5). The H-D exchange is revealed by spectroscopic results obtained in situ on the same location of BSA fibril in water and heavy water. Measured IR spectra are displayed in Supplementary Fig. S6. When in heavy water, since the majority of amide II band absorption is from $\mathrm{N}-\mathrm{H}$ bonds in proteins, the replacement of N-H with N-D bonds redshifts the amide II absorption from 1550 to $1460 \mathrm{~cm}^{-}$ ${ }^{1} \cdot{ }^{36}$ As a result, a strong amide II band of the BSA fibril is observed in heavy water, which centers around $1460 \mathrm{~cm}^{-1}$ and indicates the completion of H-D exchange.

To estimate the spatial resolution of LiPFIR imaging, a sectioned signal profile from the edge of BSA fibril is shown in Figure 3d, and a spatial resolution of $8 \mathrm{~nm}$ is obtained for LiPFIR in water. By comparison, contact mode AFM-IR in water recently achieved a $20 \sim 25 \mathrm{~nm}$ resolution, ${ }^{11,}{ }^{12}$ from a 100-nm spatial resolution in its early implementation. ${ }^{7}$ 


\section{Investigating Yeast Budding Site in the Liquid Phase}

Avoiding the destructive shear force means that the LiPFIR microscopy is suitable for imaging highly uneven soft matter samples with large height variations in the water, such as biological structures from cells. To demonstrate the capacity of LiPFIR in imaging large and soft biological samples, we use it to examine the chemical composition of large cell wall particles derived from the yeast Saccharomyces cerevisiae. These cell wall particles are often referred to as zymosan particles, which represent a group of naturally occurring immunomodulators for therapeutic applications. $^{37-39}$

Zymosan particles exhibit different shapes in the air and the aqueous phase. Figure 5a shows the topography of a dried zymosan particle in the air. The dried zymosan particle exhibits a flattened disk-like shape with an average height of $\sim 250 \mathrm{~nm}$. In the dried form, the nucleus of the original yeast becomes noticeable and appears protruded from the center of the zymosan particle. ${ }^{40,41}$ Another noticeable feature on the zymosan particle is the presence of a bud scar on the lower right. The bud scar is a ring-shaped fracture formed during the budding (division) process of yeast cells. We first perform air-phase peak force IR microscopy on this zymosan particle. The sample is illuminated at $1030 \mathrm{~cm}^{-1}$ (C-O-C vibrations) and $1630 \mathrm{~cm}^{-1}$ (amide I band), resolving the spatial distribution of polysaccharides and proteins spatially. ${ }^{42}$ As shown in Figure 5b-c, the IR image at $1030 \mathrm{~cm}^{-1}$ suggests that polysaccharide components, which mainly included $\beta$-glucan and mannan, are evenly distributed across the zymosan surface. In comparison, the IR image at $1630 \mathrm{~cm}^{-1}$ clearly shows the uneven distribution of proteins. Proteins are enriched in multiple scattered spots, the nucleus, and the bud scar. Other locations of the zymosan particle also exhibit a small IR signal at $1630 \mathrm{~cm}^{-1}$, indicating proteins are present together with polysaccharides on the rest area of the zymosan surface. 
Dried zymosan particles, after immersed in heavy water, restore to their native ellipsoidal shape. Figure 5d shows the topography of two zymosan particles in heavy water with heights of more than $1 \mu \mathrm{m}$. The height of zymosan particles increases from $200 \sim 300 \mathrm{~nm}$ in the air to up to $\sim 1.8$ $\mu \mathrm{m}$ in heavy water, and the large nucleus observed in the air disappears because it is now enclosed inside the shell of the zymosan particle. An unusual aspect of the AFM image of Figure 5d is that it captures two zymosan particles in the budding process with a septum formed in between. LiPFIR images are displayed in Figure 5e-f. In Figure 5e, an even distribution of polysaccharides is observed. In Figure 5f, the distribution of proteins also appears uniform, and slightly stronger signals present at peripherals, because the evanescent IR field is stronger at lower heights from the $\mathrm{Ge} /$ water interface. Interestingly, strong $1630 \mathrm{~cm}^{-1}$ IR absorptions that correspond to amide I are observed on a cluster of material close to the budding site, as indicated by the arrow in Figure $5 f$. The cluster is connected with the budding septum from the bottom, as revealed by the LiPFIR images. The measurement suggests that the septum in the budding process contains molecules of amide I band, e.g., protein or chitin. ${ }^{43}$

To further confirm the chemical composition of the cluster in the vicinity of the budding site, four point spectra are collected and shown in Figure 5g. Positions 1 and 3 are taken on the two zymosan particles, and position 2 is on the septum. Locations 1-3 show similar IR signatures of polysaccharides: The peak at $1190 \mathrm{~cm}^{-1}$ is attributed to the absorption of heavy water inside the zymosan particles; small IR absorptions between $1000 \sim 1100 \mathrm{~cm}^{-1}$ and the peak at $1220 \mathrm{~cm}^{-1}$ are due to the $\mathrm{C}-\mathrm{O}$ stretching, and the peak at $1430 \mathrm{~cm}^{-1}$ is attributed to $\mathrm{C}-\mathrm{H}$ bending. The spectrum collected on position 4 , which is the bright spot observed at $1630 \mathrm{~cm}^{-1}$, however, does not show discernable signatures of polysaccharide and chitin, which absorb around $1000 \sim 1100 \mathrm{~cm}^{-1}$. Instead, 
a sole amide I band around $1630 \mathrm{~cm}^{-1}$ is observed. This finding confirms that the highlighted cluster observed at $1630 \mathrm{~cm}^{-1}$ is mainly composed of proteins, and a medium band around 1430 $\mathrm{cm}^{-1}$ is attributed to the H-D exchanged amide II band. The proteins in the cluster may participate in the synthesis of chitins during the budding process, as suggested by previous studies. ${ }^{44}$

\section{Discussion}

The ability of LiPFIR represents an advancement for nanoscale IR microscopy. The nondestructiveness, label-free imaging capability of LiPFIR enables the in situ measurement of the physical transformations and chemical reactions at the solid/liquid interface. Wide varieties of industrially significant chemical reactions happen in the fluid phase, where the mass transportation capabilities are more favorable than that in the air phase. Almost all biological processes at the molecular level take place in the aqueous conditions, where the biomolecules are in their native state. The LiPFIR microscopy can extend the standard PFIR into these two important areas of research.

The non-destructiveness of LiPFIR stems from the precise control of the maximal tip-sample forces in the PFT feedback. In the liquid phase, intermolecular forces between the solvent molecule and sample molecule destabilize the sample surface, making the surface more vulnerable to mechanical scratches by the AFM tip than in the air phase. To preserve the integrity of the sample surface, the lateral shear force between the AFM tip and sample should be minimized. In the PFT mode, the AFM tip only momentarily contacts with the sample surface for tens of $\mu$ s for every PFT cycle. The shear force only builds up momentarily during the contact and is released when the tip-sample detaches in each PFT cycle. No large lateral shear force is present. Consecutive measurements with LiPFIR over an extended period ( $>8$ hours) on the delicate sample of protein 
fibrils do not induce any noticeable changes in the topography or disruptions of the sample surface (Supplementary Figure S5). In the zymosan studies of Figure 5, the zymosan particle filled with heavy water exhibit a height of $1.8 \mu \mathrm{m}$ over a lateral dimension of $5 \mu \mathrm{m}$. The zymosan particle is also very soft, with a measured reduced Young's modulus of only $0.1 \mathrm{MPa}$ in heavy water (Supplementary Figure S7). As an analogy, the zymosan in the liquid phase behaves like a waterfilled balloon and the nanoscopic AFM tip acts as a needle. Scratching of the needle on the waterfilled balloon would easily break it. This is a type of sample that would be challenging for the contact mode AFM. The cantilever deflection setpoint should be very small to reduce the lateral force; the gain of the AFM feedback loop has to be large to cope with the large height changes. However, a large feedback gain based on a low set point would amplify noise in the AFM scan, leading to possible sample damages when the AFM tip is out of topography feedback. In the LiFPIR, we use a high tapping amplitude of $100 \mathrm{~nm}$ and a low peak force setpoint of $1 \mathrm{nN}$ to measure the zymosan sample. It provided satisfactory topography feedback and IR imaging capability.

The LiPFIR microscopy provides a spatial resolution of $<8 \mathrm{~nm}$, which is on par with the air-phase PFIR microscopy. In comparison, the state-of-the-art resonantly enhanced contact mode AFM-IR techniques (i.e., REINS and resonantly enhanced PTIR) can achieve $20 \sim 25 \mathrm{~nm}$ spatial resolution in the liquid. ${ }^{11,} 12$ LiPFIR gives a $2.5 \sim 3$ times improved spatial resolution for liquid-phase IR microscopy. In comparison, the recent development of total internal reflection scattering-type scanning near-field optical microscopy (s-SNOM) in the liquid that achieves a spatial resolution of $50 \mathrm{~nm}$ in a line scan with the first-order harmonic demodulation. ${ }^{14}$ Also, in order for s-SNOM to conveniently deliver IR imaging capability, a narrowband light source has to be used. ${ }^{15}$ On the other hand, performing spectroscopy with narrow-band light sources in s-SNOM for 
interferometric detection is time-consuming. To obtain both IR imaging and spectroscopy for sSNOM, both broadband and narrowband IR sources are often required. In comparison, both LiPFIR and the contact mode AFM-IR can readily acquire both 2D IR images and point spectra without the need for interferometric detection.

The detection of photothermal expansions through lock-in detection of the cantilever oscillation is the core mechanism of contact mode AFM-IR and PiFM. However, the high hydrodynamic drag force from the fluid significantly reduces the $\mathrm{Q}$ factor of the cantilever. Consequently, the signal transduction capability is compromised. In contrast, the LiPFIR does not require lock-in detection. The cantilever deflections are detected during the short period of the tip-sample contact regime in the PFT cycle. Therefore, the lack of the high Q factor of the cantilever in fluid affects the LiPFIR microscopy much less than it affects the contact mode AFM-IR or PiFM, if implemented in the fluid phase.

\section{Conclusion}

The compatibility with liquid-phase environments, the high spatial resolution, and the chemical sensitivity enable the LiPFIR microscopy to be a versatile platform for monitoring transformations at solid/liquid interfaces, non-intrusively, and label-free. The LiPFIR microscope with the fluid perfusion chamber permits the nano-IR imaging and spectroscopy on spatial locations of interest under different liquid compositions that cause physical transformation and/or chemical reactions. This capability allows for in situ and in operando studying a broad range of solid/liquid interfacial chemical and biological processes, such as heterogeneous chemical reactions and catalysis, ${ }^{45}$ polymer membranes for biofouling mitigation, ${ }^{46}$ drug-protein interactions, ${ }^{47}$ as well as chemical sensing through mid-IR polaritons. ${ }^{48}$ 


\section{Methods and Materials}

\section{LiPFIR apparatus}

To synchronize laser pulses with peak force tapping (PFT), a waveform of PFT frequency was

routed out from an AFM controller (Nanoscope V, Bruker). This waveform was then amplified and connected to a lock-in amplifier (MFLI, Zurich Instruments), which generates a TTL waveform at the same PFT frequency. The TTL waveform was used to trigger a function generator (HDG2022B, Hantek) working in the burst mode, which generated a train of electric pulses that were used to trigger the QCL. The repetition rate of the pulse train was modified by changing parameters of the function generator, and the duration (which is proportional to the energy) of each laser pulse was controlled by the QCL program. The laser pulse duration used in this study ranged from 100-1000 ns (the energy of each pulse corresponds to 20-200 nJ), depending on the signal strength and quality. The cantilever deflection signal is acquired by a digitizer (PXI-5122, National Instruments) and processed through a program written in LabVIEW (National Instruments) in realtime. Analog outputs from the LabVIEW program were routed into the AFM controller to simultaneously form IR images. In spectroscopy mode, the scan size is set to zero so that the tip is parked on the spot of measurement. The deflection waveform was recorded along with the laser frequency. The data was then processed by MATLAB to produce an IR spectrum.

The laser repetition rate for LiPFIR measurements shown in the manuscript was adjusted case by case, as the contact resonance of the cantilever is affected by many factors, including the type of AFM cantilever, the sample itself, and tapping conditions. In addition, since multiple contact resonances of the cantilever are present in the aqueous phase, one needs to choose the appropriate contact resonance that provides the best signal quality. For most LiPFIR experiments, Au-coated 
AFM tips (HQ:NSC19/Cr-Au, MikroMasch) with the end radius of around $25 \mathrm{~nm}$ and 10-20 laser pulses were used. For the measurement of polymer blends in heavy water, the pulse repetition rate of the pulse train was tuned to $123 \mathrm{kHz}$. The LiPFIR signal was obtained from the FFT peak at $123 \mathrm{kHz}$, as well. For the measurement of polymer blends in ethanol, the pulse repetition rate was set as $264 \mathrm{kHz}$. The LiPFIR signal was obtained from the FFT peak at $86 \mathrm{kHz}$. For the H-D exchange of the BSA fibril, the pulse repetition rate and the FFT peak were both at $262 \mathrm{kHz}$. For measuring zymosan particles, a new Pt-coated tip (PFTUNA, Bruker) with the end radius of 25 $\mathrm{nm}$ was used, and the laser repetition rate and FFT peak were both at $210 \mathrm{kHz}$. For the measurement of the click-reaction, Au-coated tips were used. The laser repetition rate and the LiPFIR signal were both at $220 \mathrm{kHz}$. The duration of each laser pulse was adjusted between 100$1000 \mathrm{~ns}$ to optimize the imaging quality.

For all measured samples, the PFT frequency was set as $1 \mathrm{kHz}$, the peak force setpoint is set in a range of 3-15 nN, and the PFT amplitude was $100-150 \mathrm{~nm}$.

\section{Simulation and Calculation}

The simulation of the tip-enhanced evanescent field in Figure $1 \mathrm{~b}$ was done by the program Lumerical FDTD (Lumerical Inc.). In the simulation, a Au tip with an end radius of $30 \mathrm{~nm}$ was placed $3 \mathrm{~nm}$ above the germanium surface. A P-polarized (polarized in-plane, as shown in Figure 1a-b) plane wave was used to simulate the laser that generated the total internal reflection with an incident angle of $20^{\circ}$. The critical angle was calculated using Snell's law, and the field strength Ez was calculated using the formula in the literature. ${ }^{22}$

\section{Preparations of polymer blends and click chemistry}


Polystyrene (PS, Mw: 192000, Sigma-Aldrich) and polymethyl methacrylate (PMMA, Mw: 120000, Sigma-Aldrich) were dissolved in toluene to form a $12 \mathrm{mg} / \mathrm{mL}$ solution (the weight ratio of PS:PMMA is 1:1.5). Then, the blend polymer sample was made by spin-coating $50 \mu \mathrm{L}$ of the solution onto a $3-\mathrm{cm}^{2}$ top surface of Germanium prism at $2500 \mathrm{rpm}$ for $1 \mathrm{~min}$ by a spin-coat machine (KW-4A, MicroNano Tools) and a customized adaptor for the prism.

For measurements of click reactions, the germanium prism was spin-coated with a mixture in toluene of $\omega$-azide-terminated PMMA (P41623-MA-N3, Polymer Source, MW: 1000) and polystyrene (Sigma Aldrich, MW: 192000) with the concentration of $17 \mathrm{mg} / \mathrm{mL}$ and $25 \mathrm{mg} / \mathrm{mL}$, respectively. The reaction solution is made of $\mathrm{CuSO}_{4}(1 \mathrm{mM}$, prepared from copper(II) sulfate pentahydrate, Sigma Aldrich), $\mathrm{C}_{6} \mathrm{H}_{7} \mathrm{NaO}_{6}(7 \mathrm{mM}$, freshly prepared from (+)-sodium L-ascorbate, Sigma Aldrich) and biotin-PEG4-alkyne (7 mM, Sigma Aldrich). After locating a scanning position with AFM in the air, the cantilever was first retracted from the surface by $40 \mu \mathrm{m}$, and the fluid chamber is filled with a total of $40 \mu \mathrm{L}$ reaction solution by a syringe.

\section{Preparation of BSA fibrils}

Bovine serum albumin (99\%, Sigma-Aldrich) was dissolved in $1 \times$ PBS in a concentration of 10 $\mathrm{mg} / \mathrm{mL}$ and stored in a $4^{\circ} \mathrm{C}$ fridge. The BSA solution was then diluted with deionized water to 1 $\mathrm{mg} / \mathrm{mL}$. The BSA solution was kept at room temperature for a month to form BSA fibrils, and then $40 \mu \mathrm{L}$ of it was drop-casted on the Ge surface. The Germanium prism surface was functionalized with (3-aminopropyl)triethoxysilane (APTES) (Sigma-Aldrich) to immobilize proteins. The prism surface was first washed by soaking it in chloroform three times and 5 minutes for each time. Then, the prism was incubated with $45 \mu \mathrm{L}$ of APTES and $15 \mu \mathrm{L}$ of triethylamine (Sigma-Aldrich) in a $5 \mathrm{~L}$ desiccator filled with argon gas for 2 hours. After two hours, the vials 
containing APTES and triethylamine were removed, and the desiccator was sealed for another 8hour incubation.

\section{Preparation of zymosan particles}

Zymosan particles were purchased from InvivoGen (San Diego, CA). To prepare samples for LiPFIR measurements, $20 \mu \mathrm{L}$ of the aqueous solution containing zymosan particles at $20 \mu \mathrm{g} / \mathrm{mL}$ concentration was dropped and dried on the Germanium prism. In LiPFIR measurement, the sample was immersed in heavy water.

\section{Acknowledgments}

H.W. is thankful for Yuyuan Zhou for the help of the silanization of germanium prism and Wenpeng Cao for the help of providing BSA proteins. X. G. X. would like to thank the support from Beckman Young Investigator Award from the Arnold and Mabel Beckman Foundation and the Sloan Research Fellowship from the Alfred P. Sloan Foundation. H. W. and X.G.X. would also like to thank the support from the National Science Foundation, award number CHE 1847765. W. L. and Y. Y. acknowledge support from the National Institute of General Medical Sciences of NIH under Award Number R35GM124918. The content is solely the responsibility of the authors and does not necessarily represent the official views of NIH.

\section{Author contribution}

X. G. X. designed the idea of LiPFIR. H.W. and X.G.X built the experimental apparatus. H.W. performed the numerical simulation, collected the data, and performed data analysis. Q. X. prepared the sample for the click reaction measurement. H.W., J. M. G., and X. G. X. wrote the manuscript. W. L. and Y. Y. provided the zymosan sample. X. G. X guided the overall research. 


\section{References}

1. Abbe, E., Beiträge zur Theorie des Mikroskops und der mikroskopischen Wahrnehmung. Archiv für mikroskopische Anatomie 1873, 9 (1), 413-418.

2. Centrone, A., Infrared Imaging and Spectroscopy Beyond the Diffraction Limit. Annual Review of Analytical Chemistry 2015, 8 (1), 101-126.

3. Richards, D.; Zayats, A.; Keilmann, F.; Hillenbrand, R., Near-field microscopy by elastic light scattering from a tip. Philosophical Transactions of the Royal Society of London. Series A: Mathematical, Physical and Engineering Sciences 2004, 362 (1817), 787-805.

4. Dazzi, A.; Prater, C. B., AFM-IR: Technology and Applications in Nanoscale Infrared Spectroscopy and Chemical Imaging. Chemical Reviews 2017, 117 (7), 5146-5173.

5. Nowak, D.; Morrison, W.; Wickramasinghe, H. K.; Jahng, J.; Potma, E.; Wan, L.; Ruiz, R.; Albrecht, T. R.; Schmidt, K.; Frommer, J., Nanoscale chemical imaging by photoinduced force microscopy. Science advances 2016, 2 (3), e1501571.

6. Wang, L.; Wang, H.; Wagner, M.; Yan, Y.; Jakob, D. S.; Xu, X. G., Nanoscale simultaneous chemical and mechanical imaging via peak force infrared microscopy. Sci. Adv. 2017, 3 (6), e1700255.

7. Mayet, C.; Dazzi, A.; Prazeres, R.; Allot, F.; Glotin, F.; Ortega, J. M., Sub-100 nm IR spectromicroscopy of living cells. Optics letters 2008, 33 (14), 1611-1613.

8. Dazzi, A.; Prazeres, R.; Glotin, F.; Ortega, J. M., Local infrared microspectroscopy with subwavelength spatial resolution with an atomic force microscope tip used as a photothermal sensor. Optics letters $\mathbf{2 0 0 5}$, 30 (18), 2388-2390.

9. Lu, F.; Jin, M.; Belkin, M. A., Tip-enhanced infrared nanospectroscopy via molecular expansion force detection. Nature Photonics 2014, 8 (4), 307-312.

10. Jakob, D. S.; Wang, H.; Zeng, G.; Otzen, D. E.; Yan, Y.; Xu, X. G., Peak Force Infrared-Kelvin Probe Force Microscopy. Angewandte Chemie International Edition 2020, 59 (37), 16083-16090.

11. Jin, M.; Lu, F.; Belkin, M. A., High-sensitivity infrared vibrational nanospectroscopy in water. Light: Science \& Applications 2017, 6 (7), lsa201796.

12. Ramer, G.; Ruggeri, F. S.; Levin, A.; Knowles, T. P. J.; Centrone, A., Determination of polypeptide conformation with nanoscale resolution in water. ACS nano 2018, 12 (7), 6612-6619.

13. Wang, H.; Janzen, E.; Wang, L.; Edgar, J. H.; Xu, X. G., Probing Mid-Infrared Phonon Polaritons in the Aqueous Phase. Nano letters 2020, 20 (5), 3986-3991.

14. O’Callahan, B. T.; Park, K.-D.; Novikova, I. V.; Jian, T.; Chen, C.-L.; Muller, E. A.; El-Khoury, P. Z.; Raschke, M. B.; Lea, A. S., In Liquid Infrared Scattering Scanning Near-Field Optical Microscopy for Chemical and Biological Nanoimaging. Nano letters 2020.

15. Pfitzner, E.; Heberle, J., Infrared Scattering-Type Scanning Near-Field Optical Microscopy of Biomembranes in Water. The Journal of Physical Chemistry Letters 2020, 8183-8188.

16. Li, J.; Pang, J.; Yan, Z.-d.; Jahng, J.; Li, J.; Morrison, W.; Liang, J.; Zhang, Q.-Y.; Xia, X.-H. J. a. p. a., Antenna enhancing infrared photoinduced force imaging in aqueous environment with superresolution and hypersensitivity. 2020.

17. Alsteens, D.; Dupres, V.; Yunus, S.; Latgé, J.-P.; Heinisch, J. r. J.; Dufrêne, Y. F., High-resolution imaging of chemical and biological sites on living cells using peak force tapping atomic force microscopy. Langmuir 2012, 28 (49), 16738-16744.

18. Rico, F.; Su, C.; Scheuring, S., Mechanical mapping of single membrane proteins at submolecular resolution. Nano letters 2011, 11 (9), 3983-3986.

19. Rosa-Zeiser, A.; Weilandt, E.; Hild, S.; Marti, O., The simultaneous measurement of elastic, electrostatic and adhesive properties by scanning force microscopy: pulsed-force mode operation. Measurement Science and Technology 1997, 8 (11), 1333.

20. Pittenger, B.; Erina, N.; Su, C., Quantitative mechanical property mapping at the nanoscale with PeakForce QNM. Application Note Veeco Instruments Inc. 2010, 1-12. 
21. Johnson, K. L.; Woodhouse, J., Stick-slip motion in the atomic force microscope. Tribology Letters 1998, 5 (2), 155-160.

22. Milosevic, M., On the nature of the evanescent wave. Applied spectroscopy 2013, 67 (2), 126-131.

23. Wang, L.; Wagner, M.; Wang, H.; Pau-Sanchez, S.; Li, J.; Edgar, J. H.; Xu, X. G., Revealing Phonon Polaritons in Hexagonal Boron Nitride by Multipulse Peak Force Infrared Microscopy. Advanced Optical Materials 2020, 8 (5), 1901084.

24. Papanu, J.; Hess, D.; Soane, D.; Bell, A., Swelling of poly (methyl methacrylate) thin films in low molecular weight alcohols. Journal of Applied Polymer Science 1990, 39 (4), 803-823.

25. Hoogenboom, R.; Becer, C. R.; Guerrero-Sanchez, C.; Hoeppener, S.; Schubert, U. S., Solubility and Thermoresponsiveness of PMMA in Alcohol-Water Solvent Mixtures. Australian Journal of Chemistry 2010, 63 (8), 1173-1178.

26. Kolb, H. C.; Finn, M. G.; Sharpless, K. B., Click Chemistry: Diverse Chemical Function from a Few Good Reactions. Angewandte Chemie International Edition 2001, 40 (11), 2004-2021.

27. Hein, J. E.; Fokin, V. V., Copper-catalyzed azide-alkyne cycloaddition (CuAAC) and beyond: new reactivity of copper(i) acetylides. Chemical Society Reviews 2010, 39 (4), 1302-1315.

28. Meldal, M., Polymer "Clicking" by CuAAC Reactions. Macromolecular Rapid Communications 2008, 29 (12-13), 1016-1051.

29. Geva-Zatorsky, N.; Alvarez, D.; Hudak, J. E.; Reading, N. C.; Erturk-Hasdemir, D.; Dasgupta, S.; von Andrian, U. H.; Kasper, D. L., In vivo imaging and tracking of host-microbiota interactions via metabolic labeling of gut anaerobic bacteria. Nature Medicine 2015, 21 (9), 1091-1100.

30. McKay, Craig S.; Finn, M. G., Click Chemistry in Complex Mixtures: Bioorthogonal Bioconjugation. Chemistry \& Biology 2014, 21 (9), 1075-1101.

31. Zhang, W.; Zhao, Q.; Yuan, J., Porous polyelectrolytes: the interplay of charge and pores for new functionalities. Angewandte Chemie International Edition 2018, 57 (23), 6754-6773.

32. Comby, S.; Gunnlaugsson, T., Luminescent lanthanide-functionalized gold nanoparticles: exploiting the interaction with bovine serum albumin for potential sensing applications. ACS nano 2011, 5 (9), 71847197.

33. Ruggeri, F. S.; Mannini, B.; Schmid, R.; Vendruscolo, M.; Knowles, T. P. J., Single molecule secondary structure determination of proteins through infrared absorption nanospectroscopy. Nature Communications 2020, 11 (1), 2945.

34. de la Arada, I.; Seiler, C.; Mäntele, W., Amyloid fibril formation from human and bovine serum albumin followed by quasi-simultaneous Fourier-transform infrared (FT-IR) spectroscopy and static light scattering (SLS). European Biophysics Journal 2012, 41 (11), 931-938.

35. Pearce, F. G.; Mackintosh, S. H.; Gerrard, J. A., Formation of amyloid-like fibrils by ovalbumin and related proteins under conditions relevant to food processing. Journal of Agricultural and Food Chemistry 2007, 55 (2), 318-322.

36. Blout, E.; De Loze, C.; Asadourian, A., The deuterium exchange of water-soluble polypeptides and proteins as measured by infrared spectroscopy. Journal of the American Chemical Society 1961, 83 (8), 1895-1900.

37. Pan, Y.; Li, X.; Kang, T.; Meng, H.; Chen, Z.; Yang, L.; Wu, Y.; Wei, Y.; Gou, M., Efficient delivery of antigen to DCs using yeast-derived microparticles. Scientific reports 2015, 5, 10687.

38. De Smet, R.; Demoor, T.; Verschuere, S.; Dullaers, M.; Ostroff, G. R.; Leclercq, G.; Allais, L.; Pilette, C.; Dierendonck, M.; De Geest, B. G., $\beta$-Glucan microparticles are good candidates for mucosal antigen delivery in oral vaccination. Journal of controlled release 2013, 172 (3), 671-678.

39. Novak, M.; Vetvicka, V., $\beta$-glucans, history, and the present: immunomodulatory aspects and mechanisms of action. Journal of immunotoxicology 2008, 5 (1), 47-57.

40. Lombard, Y.; Giaimis, J.; Makaya-Kumba, M.; Fonteneau, P.; Poindron, P., A new method for studying the binding and ingestion of zymosan particles by macrophages. Journal of immunological methods 1994, 174 (1-2), 155-165. 
41. Jawhara, S.; Habib, K.; Maggiotto, F.; Pignede, G.; Vandekerckove, P.; Maes, E.; Dubuquoy, L.; Fontaine, T.; Guerardel, Y.; Poulain, D., Modulation of intestinal inflammation by yeasts and cell wall extracts: strain dependence and unexpected anti-inflammatory role of glucan fractions. PloS one 2012, 7 (7).

42. Li, W.; Wang, H.; Xu, X. G.; Yu, Y., Simultaneous Nanoscale Imaging of Chemical and Architectural Heterogeneity on Yeast Cell Wall Particles. Langmuir 2020, 36 (22), 6169-6177.

43. Fleet, G. H., Composition and structure of yeast cell walls. In Current Topics in Medical Mycology, Springer: 1985; pp 24-56.

44. Cabib, E.; Durán, A., Synthase III-dependent chitin is bound to different acceptors depending on location on the cell wall of budding yeast. Journal of Biological Chemistry 2005, 280 (10), 9170-9179.

45. Fechete, I.; Wang, Y.; Védrine, J. C., The past, present and future of heterogeneous catalysis. Catalysis Today 2012, 189 (1), 2-27.

46. Gunari, N.; Brewer, L. H.; Bennett, S. M.; Sokolova, A.; Kraut, N. D.; Finlay, J. A.; Meyer, A. E.; Walker, G. C.; Wendt, D. E.; Callow, M. E.; Callow, J. A.; Bright, F. V.; Detty, M. R., The control of marine biofouling on xerogel surfaces with nanometer-scale topography. Biofouling 2011, 27 (2), 137-149. 47. Ye, C.; Chi, H., A review of recent progress in drug and protein encapsulation: Approaches, applications and challenges. Materials Science and Engineering: C 2018, 83, 233-246.

48. Wang, H.; Janzen, E.; Wang, L.; Edgar, J. H.; Xu, X. G., Probing mid-infrared phonon polaritons in the aqueous phase. Nano Lett. 2020, 20 (5), 3986-3991. 
Figures

(a)

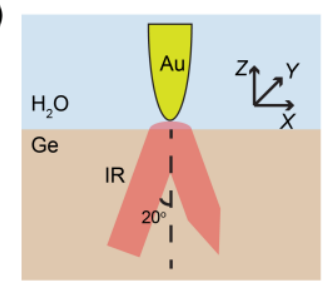

(b)

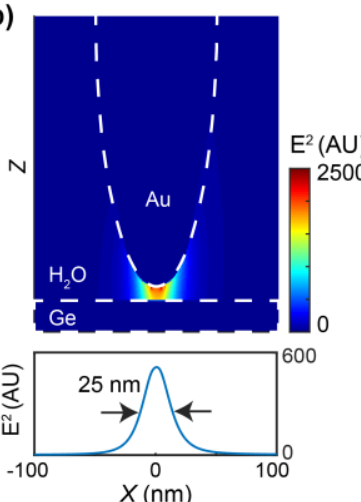

(c)

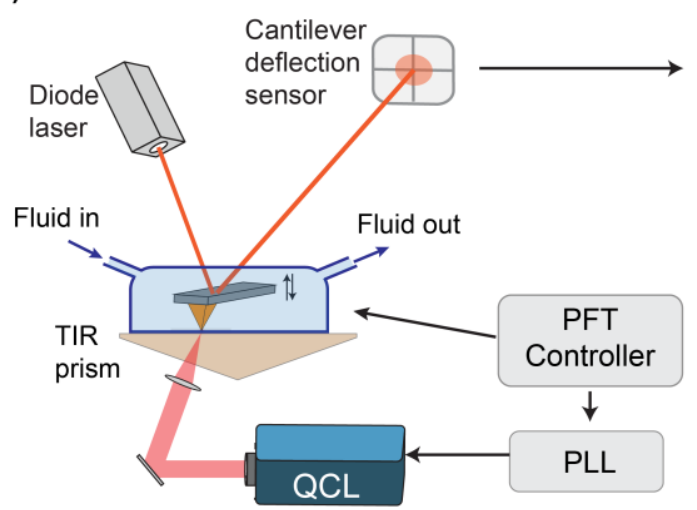

(d)
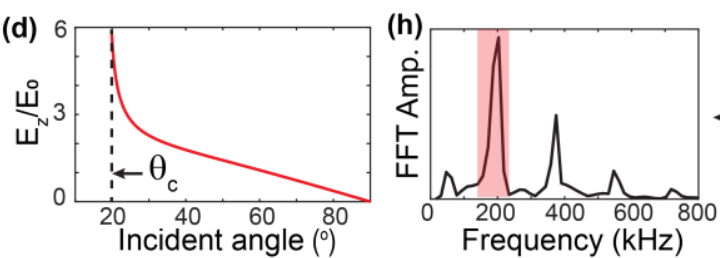

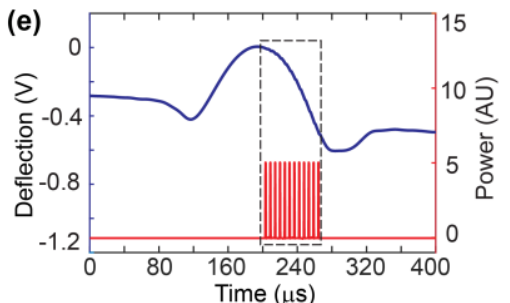

(e)

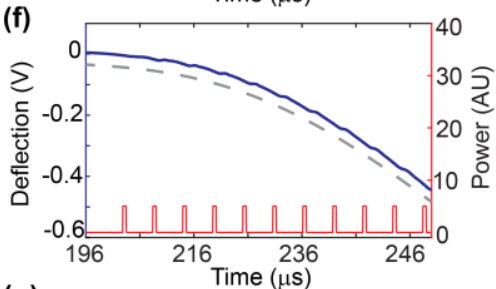

(g)

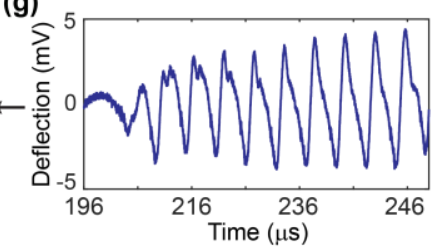

Figure 1. Design and operational principle of the LiPFIR microscopy. (a) Scheme for total internal reflection. A gold tip with an end radius of $30 \mathrm{~nm}$ is placed $3 \mathrm{~nm}$ above the Ge surface, and a p-polarized plane wave with an incident angle of $20^{\circ}$ is used to generate the evanescent field from the total internal reflection. (b) FDTD simulation of the tip-enhanced evanescent field at the $\mathrm{H}_{2} \mathrm{O} / \mathrm{Ge}$ interface. The lower panel shows the field intensity across the Ge surface. (c) Apparatus of LiPFIR microscope. (d) The relative field strength of the evanescent infrared field at the $\mathrm{Ge} /$ water interface at different incident angles. The highest field strength is achieved at the critical angle, located at $19^{\circ}$. (e) The cantilever deflection signal of PFT in water (blue). The infrared pulse train (red) is synchronized to every PFT cycle. (f) A zoomed-in region of laser-induced cantilever oscillations (blue) and infrared pulse train (red). A polynomial fit is performed on the cantilever deflection to obtain a smooth background (gray dashed curve, offset from the original position for the comparison). (g) High-frequency oscillations of the cantilever. Subtracting the background from the cantilever deflection signal isolates high-frequency oscillations due to laser-induced photothermal expansions. (h) Fast Fourier transform of (g). Usually, the strongest peak in FFT is integrated and used as the LiPFIR signal. 
(a)

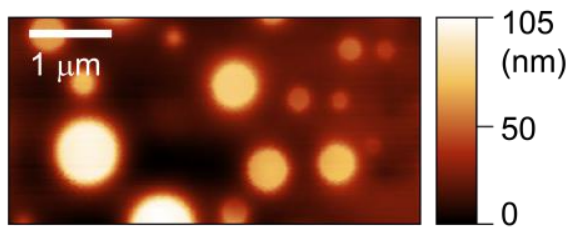

(b)

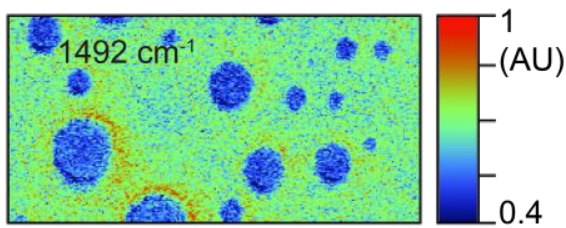

(c)

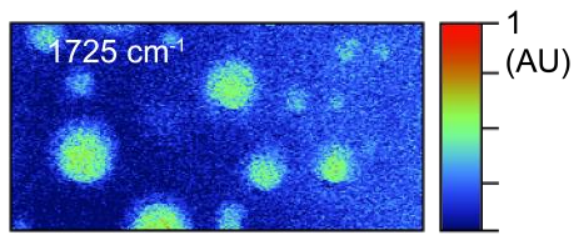

(d)

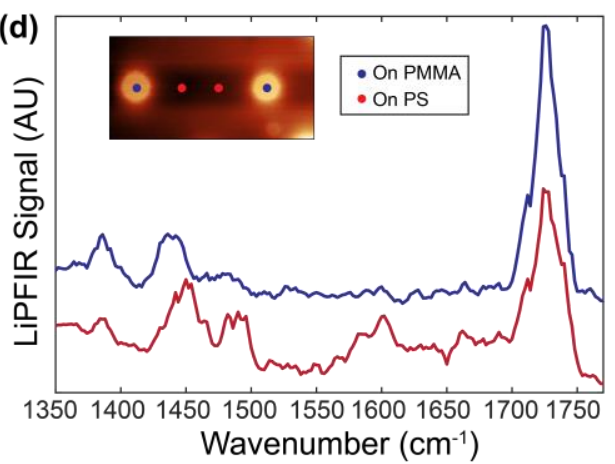

(e)

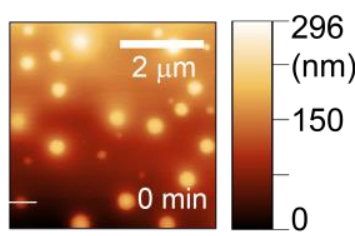

(f)

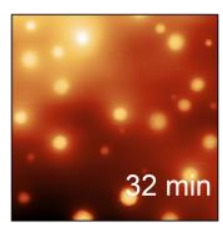

(g)

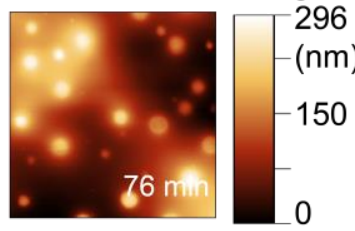

(h)

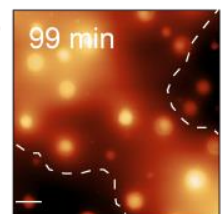

(i)

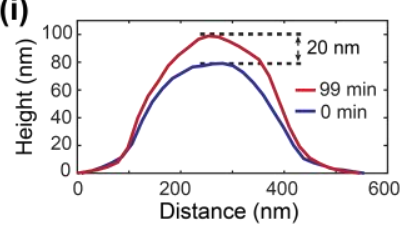

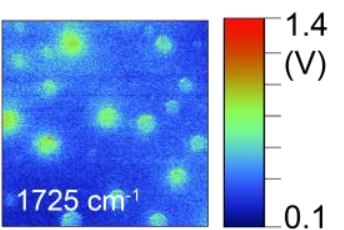
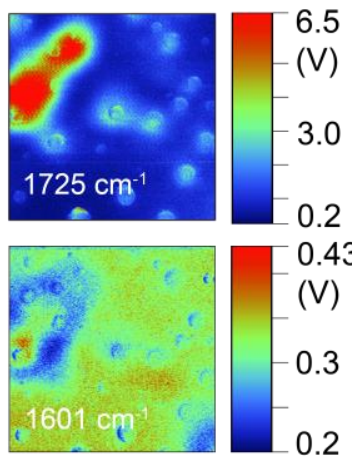

296

(nm)

150

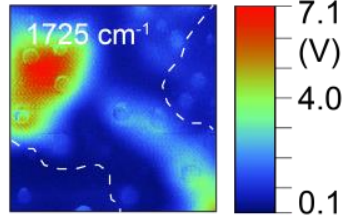

(j)

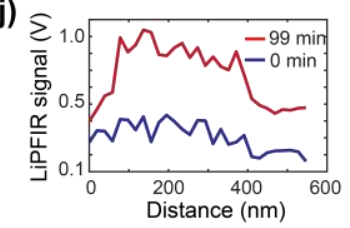

Figure 2. LiPFIR imaging and spectroscopy on PS:PMMA blend films. (a) AFM topography of a film of PS:PMMA blend in heavy water. (b-c) LiPFIR images at $1492 \mathrm{~cm}^{-1}$ (PS absorption) and $1725 \mathrm{~cm}^{-1}$ (PMMA absorption). (d) LiPFIR spectra collected from multiple locations on the PS:PMMA blend film. Inset shows the topography of a $3 \times 1.5 \mu \mathrm{m}^{2}$ area, where measuring locations are labeled as points. Two measurements on each domain were made and averaged spectra are shown. Spectra are offset from each other for a better comparison. Characteristic PS and PMMA absorption lines are marked by red and blue dashed lines, respectively. (e-h) Topography (left column) and LiPFIR (right column) images of PS:PMMA film in ethanol. Images were continously captured at $0 \mathrm{~min}, 32 \mathrm{~min}, 76 \mathrm{~min}$ and $99 \mathrm{~min}$, respectively. (i) Height profiles of a PMMA domain at $0 \mathrm{~min}$ and $99 \mathrm{~min}$. The extraction location is marked by white lines in (e) and (h). (j) LiPFIR signal profiles of the same PMMA domain. 


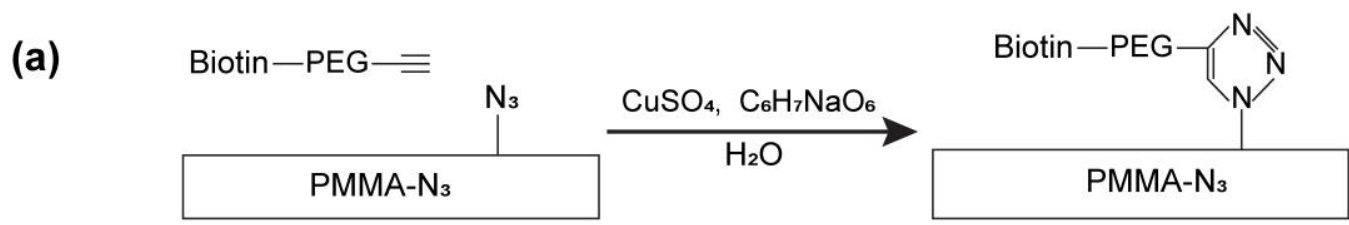

(b)
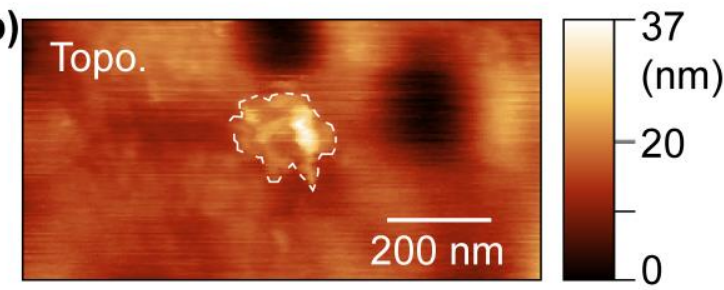

(d)

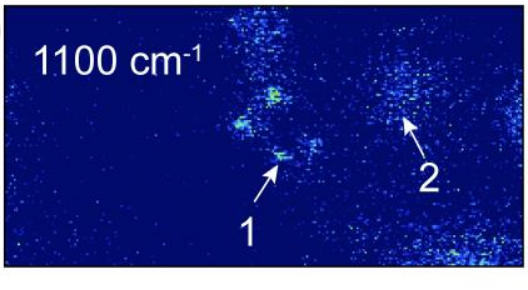

(f)

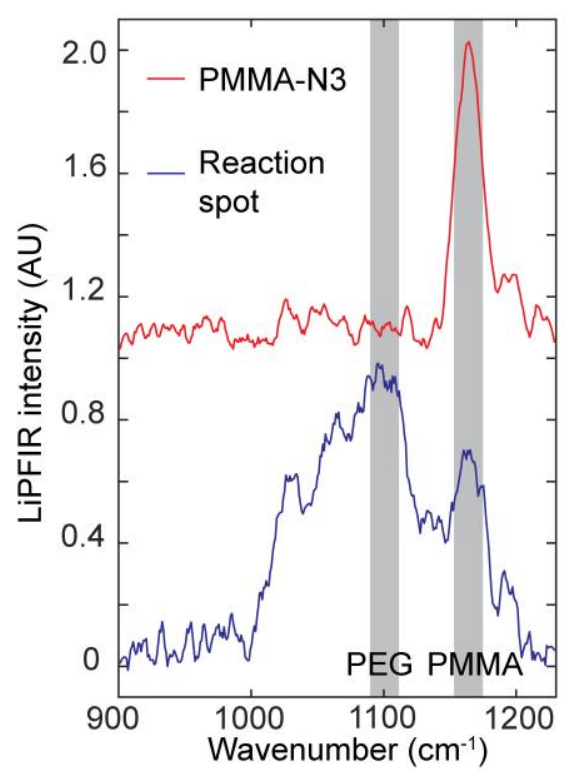

(c)
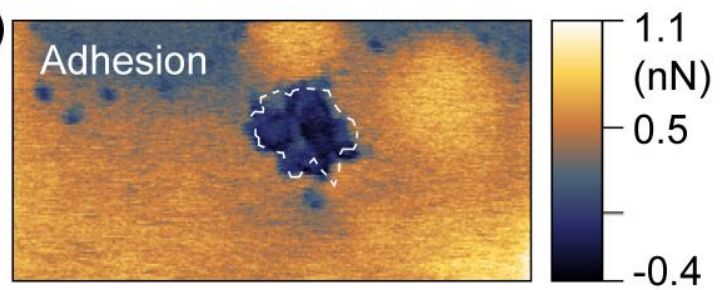

(e)
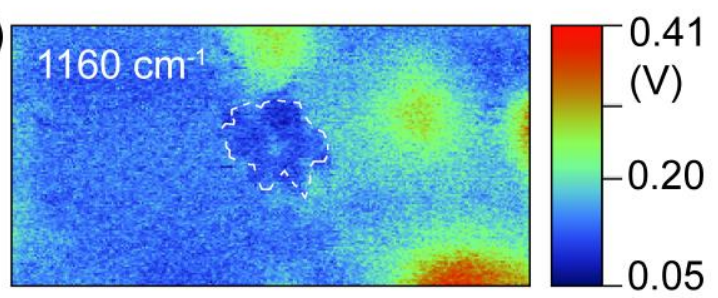

(g)

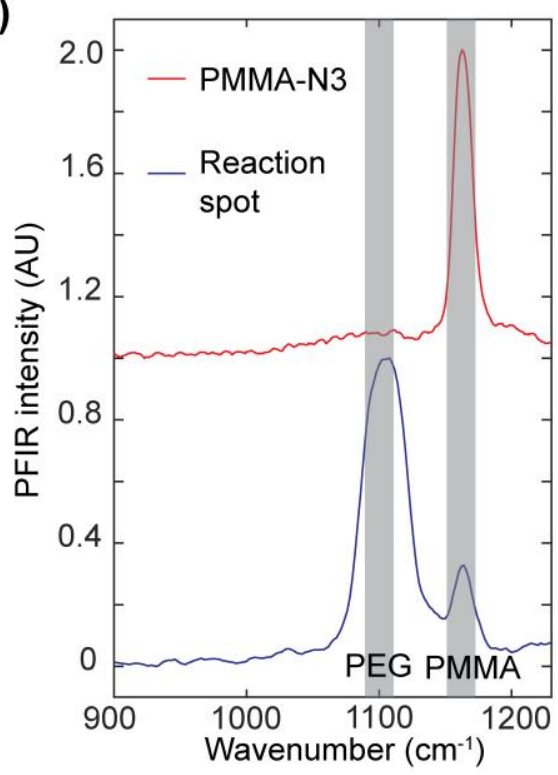

Figure 3. Revealing the click reaction site by LiPFIR. (a) Schematic of the click reaction between the alkyne-PEG-biotin in solution and the PMMA-N3 film on the germanium prism. (b) Topography of the reaction site on the PMMA-N3 film. A protruded region can be observed and is enclosed by white dashed lines. (c) Adhesion map of the same area. (d-e) LiPFIR images at 1100 $\mathrm{cm}^{-1}$ and $1160 \mathrm{~cm}^{-1}$, respectively. (f) Point spectra measured from the reaction spot (blue, position 1 in d) and the PMMA-N3 domain (red, position 2 in d). (g) Point spectra measured from the reaction spot and the PMMA-N3 domain in the air. 
(a)

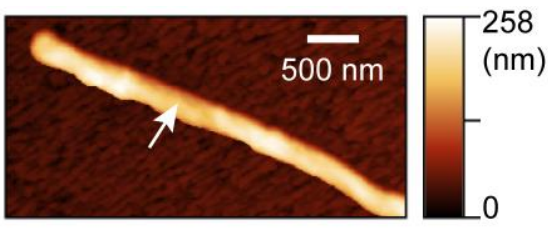

(b)

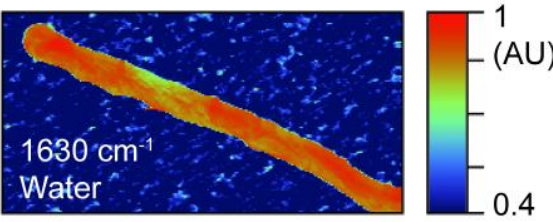

(c)

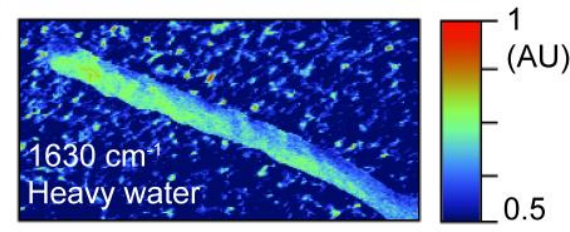

(d)

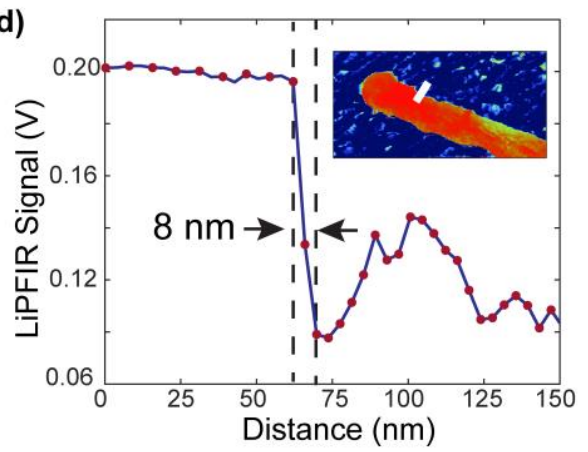

Figure 4. LiPFIR imaging of a BSA fibril in water and heavy water. (a) Topography of a 150$\mathrm{nm}$ high and 350-nm wide BSA fibril. (b) Infrared images of the BSA fibril in aqueous $0.1 \times \mathrm{PBS}$ solution at $1630 \mathrm{~cm}^{-1}$. (c) Infrared images of the same BSA fibril in heavy water at $1630 \mathrm{~cm}^{-1}$.(d) Signal profile from a high-resolution infrared image of the BSA fibril in water at $1630 \mathrm{~cm}^{-1}$ shown as the inset. A spatial resolution of $8 \mathrm{~nm}$ is obtained from the lateral distance between the signal maximum and minimum across the sharp edge of the fibril. 

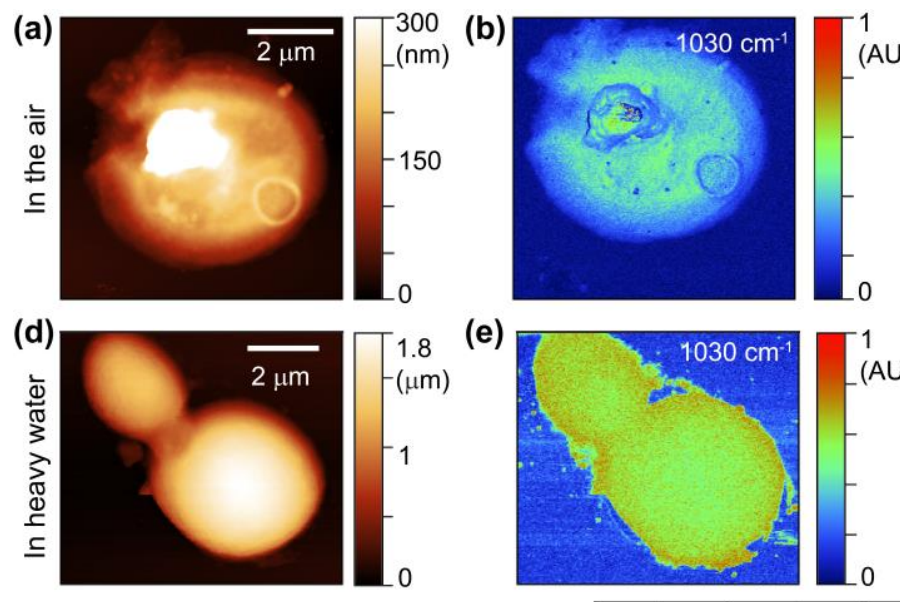

(e)

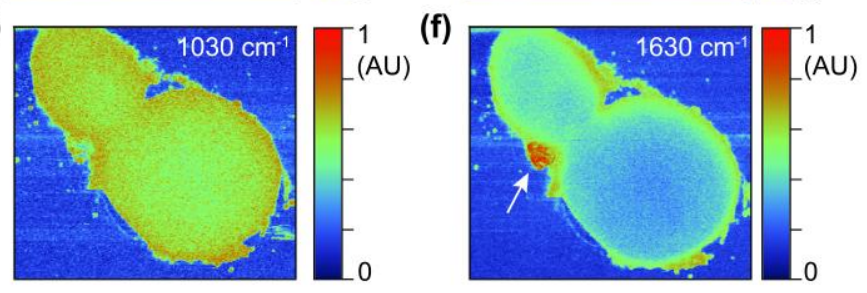

(g)
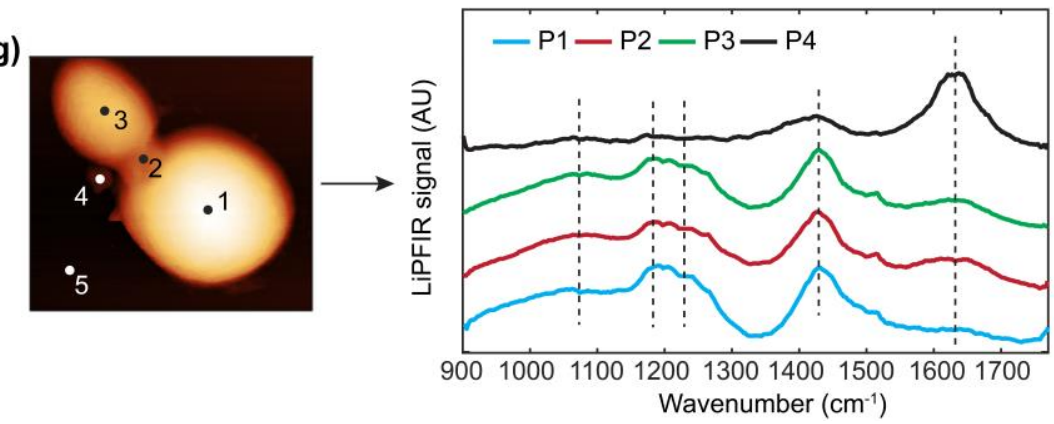

Figure 5. LiPFIR imaging and spectroscopy on zymosan particles. (a) Topography of a zymosan particle in the air. The scale bar is cut off at $300 \mathrm{~nm}$ to provide better image contrast for the bud scar of low height. (b-c) Standard PFIR images of the zymosan particle in the air at 1030 $\mathrm{cm}^{-1}$ and $1630 \mathrm{~cm}^{-1}$, respectively. (d) Topography of two zymosan particles in heavy water. (e-f) LiPFIR images of two zymosan particles in heavy water at $1030 \mathrm{~cm}^{-1}$ and $1630 \mathrm{~cm}^{-1}$, respectively. (g) The left panel indicates four spots of measurement for point spectra (positions 1 to 4). Position 5 is used to collect the baseline. The right panel shows collected spectra from four different locations on the two zymosan particles. Peaks at 1080, 1190, 1220, 1430 and $1630 \mathrm{~cm}^{-1}$ are labeled with dashed lines. 